\title{
Comparison of cytosine base editors and development of the BEable-GPS database for targeting pathogenic SNVs
}

\author{
Ying Wang ${ }^{1 \dagger}$, Runze Gao ${ }^{2,3,4 \dagger}$, Jing Wu ${ }^{2 \dagger}$, Yi-Chun Xiong ${ }^{1}$, Jia Wei ${ }^{1}$, Sipin Zhang ${ }^{2}$, Bei Yang ${ }^{5}$, Jia Chen ${ }^{2,3^{*}}$ and
} Li Yang ${ }^{1,2^{*}}$ (i)

\begin{abstract}
A variety of base editors have been developed to achieve C-to-T editing in different genomic contexts. Here, we compare a panel of five base editors on their C-to-T editing efficiencies and product purity at commonly editable sites, including some human pathogenic C-to-T mutations. We further profile the accessibilities of 20 base editors to all possible pathogenic mutations in silico. Finally, we build the BEable-GPS (Base Editable prediction of Global Pathogenic SNVs) database for users to select proper base editors to model or correct disease-related mutations. The in vivo comparison and in silico profiling catalog the availability of base editors and their broad applications in biomedical studies.
\end{abstract}

Keywords: Base editing, Base editor, CRISPR/Cas, Cytidine deaminase, Pathogenic mutation

\section{Background}

A number of base editors (BEs) [1-6], which combine different APOBEC (apolipoprotein B mRNA editing enzyme, catalytic polypeptide-like)/AID (activation-induced deaminase) cytidine deaminase family members $[7,8]$ with distinct CRISPR/Cas proteins $[9,10]$, have been developed to achieve programmable C-to- $\mathrm{T}$ changes in different sequence contexts or backgrounds. Distinct to Cas nucleases, which trigger homologydirected repair (HDR)-mediated gene correction by cleaving DNA double strands, BEs induce base changes in targeted genomic regions independent of the generation of DNA double-strand breaks (DSB) generally. Guided by the Cas moiety, BEs catalyze direct C-to-T changes with its fused cytidine deaminase moiety. A uracil DNA glycosylase inhibitor (UGI) is fused to BEs to prevent unintended mutagenesis during the process of

\footnotetext{
* Correspondence: chenjia@shanghaitech.edu.cn; liyang@picb.ac.cn

${ }^{\dagger}$ Ying Wang, Runze Gao and Jing Wu contributed equally to this work.

${ }^{2}$ School of Life Science and Technology, ShanghaiTech University, Shanghai 201210, China

${ }^{1}$ CAS Key Laboratory of Computational Biology, CAS-MPG Partner Institute for Computational Biology, Shanghai Institute of Nutrition and Health, Shanghai Institutes for Biological Sciences, University of Chinese Academy of Sciences, Chinese Academy of Sciences, Shanghai 200031, China

Full list of author information is available at the end of the article
}

base editing [1, 2], and additional UGIs co-expressed in trans with $\mathrm{BEs}$ (enhanced $\mathrm{BE}, \mathrm{eBE}$ ) further enhance the efficiency and fidelity of base editing [11]. BEs hold the potential to be used for correcting and creating pathogenic point mutations (Fig. 1a) [12-14]. However, BEs with different Cas proteins, e.g., Cas9 or Cas12a (also known as Cpf1), and different deaminases, e.g., rat APOBEC1 (rA1) or human APOBEC3A (hA3A), have not been directly compared for their utility in creating or correcting pathogenic point mutations. More importantly, a database comprehensively cataloging pathogenic point mutations that can be corrected or created by different BEs has been lacking. In this study, we experimentally compare a panel of five BEs for their editing efficiency and product purity at sites of human pathogenic C-to- $\mathrm{T}$ mutations that can be created or corrected by the same panel of BEs. We further profile the accessibilities of 20 BEs to all reported human pathogenicrelated $\mathrm{T}$-to- $\mathrm{C}$ or $\mathrm{C}$-to- $\mathrm{T}$ point mutations in silico and build a BEable-GPS (Base Editable prediction of Global Pathogenic SNVs) database to provide a resource for potential gene therapies and biomedical studies.

(c) The Author(s). 2019 Open Access This article is distributed under the terms of the Creative Commons Attribution 4.0 International License (http://creativecommons.org/licenses/by/4.0/), which permits unrestricted use, distribution, and reproduction in any medium, provided you give appropriate credit to the original author(s) and the source, provide a link to the Creative Commons license, and indicate if changes were made. The Creative Commons Public Domain Dedication waiver (http://creativecommons.org/publicdomain/zero/1.0/) applies to the data made available in this article, unless otherwise stated. 
a

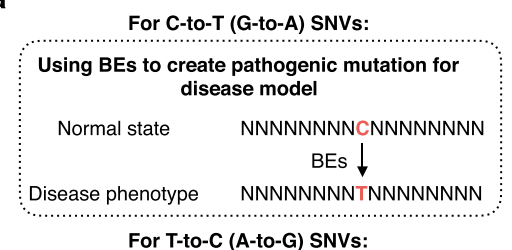

Using BEs to correct pathogenic mutation for disease treatment

Disease phenotype NNNNNNNNCNNNNNNNN BEs $\downarrow$ Normal state NNNNNNNNTNNNNNNNN

c \begin{tabular}{|c|c|c|c|c|c|c|c|c|c|c|}
\hline G C A T A C T A G G C C A T A G C T G A C G G & $\frac{\text { Preferentially editable SNVs: }}{\text { only one C in the editing window }}$
\end{tabular}

d
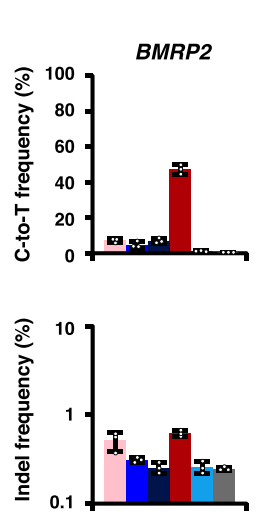

e
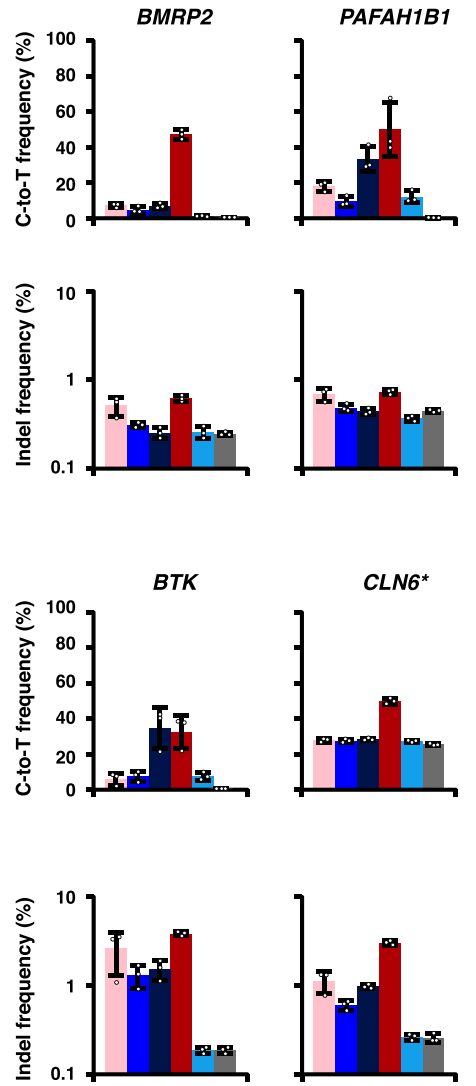

b
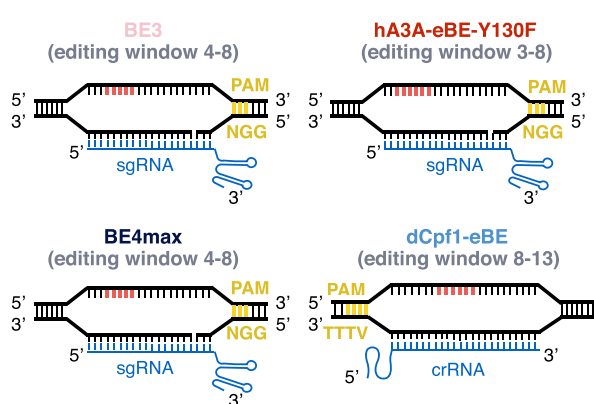

dCpf1-eBE (editing window 8-13)

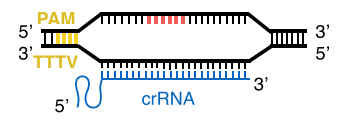

eBE-S3
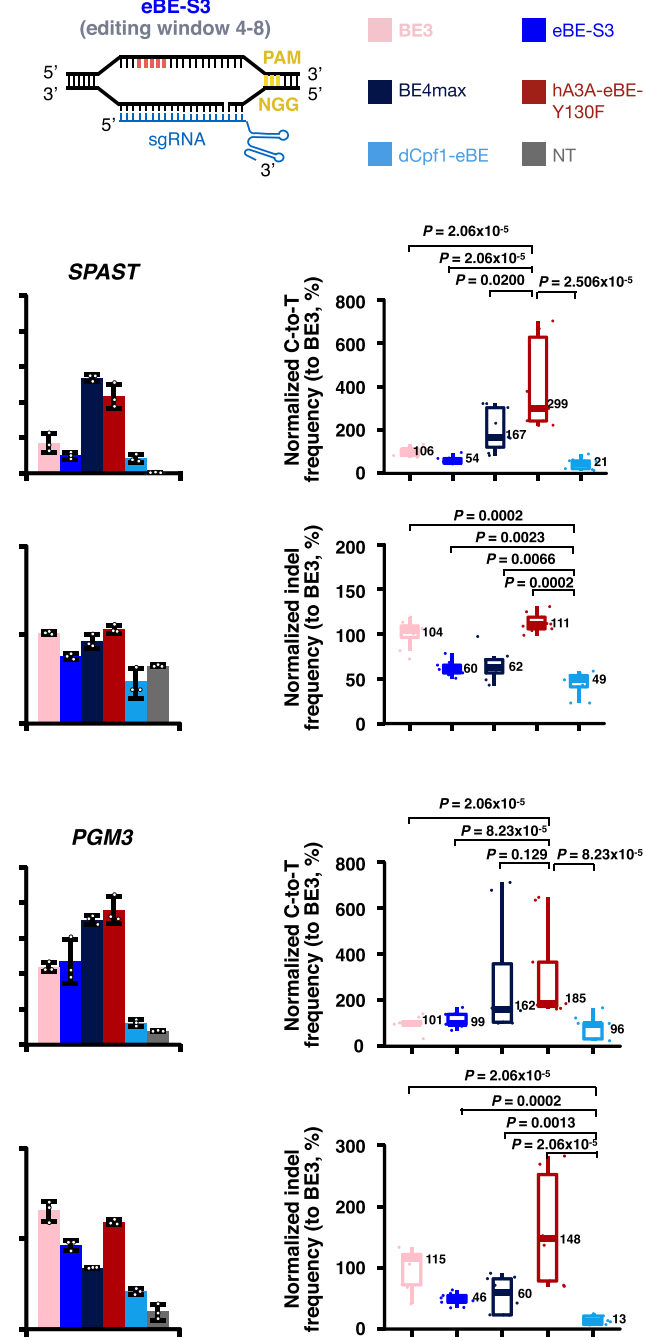

Fig. 1 Comparison of base editing outcomes at pathogenic SNVs. a The diagram to illustrate the use of BEs in creating or correcting C-to-T (G-toA) SNVs or T-to-C (A-to-G) SNVs to model or correct disease-related mutations. $\mathbf{b}$ The detailed target sites and editing windows of BE3, BE4max, eBE-S3, hA3A-eBE-Y130F, and dCpf1-eBE are shown. The cytosines were counted with the base distal to the PAM setting as position 1 in Cas9based BEs and with the base proximal to the PAM setting as position 1 in Cpf1-based BEs. c The diagram to illustrate preferentially targetable SNVs. $\mathbf{d}$ Comparison of base editing outcomes at three pathogenic SNVs that can be created by five examined BEs in 293FT cells. e Comparison of base editing outcomes at three ABEmax-created pathogenic SNVs that can be corrected by five examined BEs in 293FT cells. The C-to-T editing (top) and indel (bottom) frequencies were individually shown at target sites in $\mathbf{d}$ and $\mathbf{e}$. Asterisk, the T-to-C mutation created by ABEmax at the CLN6 gene locus is heterozygous, indicated by the high basal level of C-to-T correction by non-transfected control in e. NT, nontransfected. Data are shown as mean \pm s.d. from three independent experiments. Statistical analysis of normalized C-to-T editing frequencies and indel frequencies at these three pathogenic SNV sites is accordingly shown in the right panel in $\mathbf{d}$ and $\mathbf{e}$. Setting the ones induced by BE3 as 100\%. $P$ value, one-tailed Wilcoxon rank sum test. The median and interquartile range (IQR) are shown 


\section{Results and discussion}

The combination of different cytidine deaminases with distinct Cas proteins extends the scope of base editing in different sequence contexts or backgrounds; however, it also results in variable targeting preferences, which hampers the direct comparison of BEs. To solve this problem, we selected five BEs, including BE3 [1], eBE-S3 [11], BE4max [15], hA3A-eBE-Y130F [6], and dCpf1-eBE [5], to compare their base editing efficiency and product purity at the same genomic target sites. These selected five BEs have similar widths of editing window ( $5 \mathrm{bp}$ ) for comparison (Fig. 1b).

At three previously reported target sites [5] that can be edited by all five BEs, BE4max and hA3A-eBE-Y130F induced higher $\mathrm{C}$-to- $\mathrm{T}$ editing frequencies than the other examined BEs in 293FT cells (Additional file 1: Figure S1a, b), while hA3A-eBE-Y130F also exhibited slightly higher indel frequencies (Additional file 1: Figure S1a, c). The relatively high indels induced by hA3A-eBE-Y130F are likely caused by the high cytidine deamination activity of its hA3A moiety $[16,17]$. Although showing the lowest editing frequencies among all five tested BEs, dCpf1-eBE induced fewer indels and non-C-to- $\mathrm{T}$ conversions than the other BEs did (Additional file 1: Figure S1a, c) and therefore yielded purer editing products (Additional file 1: Figure S1d). Assumedly, the catalytically dead Cpf1 moiety in $\mathrm{dCpf1-eBE}$ makes its low editing frequency but high product purity (Additional files 3, 4, 5, and 6).

We next sought to compare the performance of these BEs to create human pathogenic C-to-T SNVs. Among reported pathogenic C-to-T SNVs [18], we selected three sites, at which all five BEs have overlapping editing windows (Additional file 1: Figure S2a). Importantly, the cytosine in each of the three selected sites is the only cytosine in the editing window, referred to as preferentially editable SNVs (Fig. 1c). Theoretically, the C-to-T conversions at these three target sites could be used to mimic human genetic disorders (Additional file 1: Figure S2b). At these sites, BE4max and hA3A-eBE-Y130F also induced higher levels of editing frequencies than the other examined BEs in 293FT cells (Fig. 1d, top), consistent with the results obtained at non-pathogenic target sites (Additional file 1: Figure S1). Notably, only hA3A-eBE-Y130F yielded efficient base editing at the loci of BMRP2 (Fig. 1d, top), while no obvious editing was induced by the other BEs. The indel frequencies induced by dCpf1-eBE were lower than those induced by the other BEs (Fig. 1d, bottom). Meanwhile, the C-to- $\mathrm{T}$ fraction induced by $\mathrm{dCpf1-eBE}$ was significantly higher than those by the other BEs (Additional file 1: Figure S2c), showing that dCpf1-eBE yielded purer editing products.

Another important application of BEs is to correct pathogenic mutations, which theoretically could be used in preclinic or clinic studies [19]. To test base editing efficiency and precision in correcting pathogenic mutations of these
BEs, we took advantage of ABEmax [15] to first create Tto- $\mathrm{C}$ mutations and then to correct them by the aforementioned five BEs (Additional file 1: Figure S3). Three reported pathogenic T-to-C/A-to-G SNV sites that can be preferentially corrected by all five BEs were selected for correction study (Additional file 1: Figure S3a, b). These pathogenic T-to-C/A-to-G mutations were generated by ABEmax individually in 293FT cells (Additional file 1: Figure S3c), and single-colony-derived cell lines with corresponding T-to-C mutations were further confirmed by Sanger sequencing (Additional file 1: Figure S3d). These Tto-C/A-to-G mutations that mimic pathogenic SNVs were further corrected by five tested BEs. As shown in Fig. 1e (top), BE4max and hA3A-eBE-Y130F induced higher efficiencies than the other examined BEs. Notably, only hA3AeBE-Y130F yielded efficient base editing at the loci of CLN6 (Fig. 1e, top), while the others induced editing similar to the background level. As expected, dCpf1-eBE induced purer editing products than the other BEs though it induced low levels of C-to-T correction efficiency (Fig. 1e, bottom and Additional file 1: Figure S4).

We further compared three representative BEs, including hA3A-eBE-Y130F with the highest editing efficiency, $\mathrm{dCpf1-eBE}$ with the purest editing product, and eBE-S3 with intermediate editing efficiency and product purity (Fig. 1d, e and Additional file 1: Figure S1), at additional sites for their editing efficiencies and product purities. Of note, these three selected BEs all express three extra copies of free UGI to enhance editing performance. As expected, hA3A-eBE-Y130F induced the highest editing frequency and $\mathrm{dCpf1-eBE}$ yielded the purest C-to-T editing product (Additional file 1: Figure S5, S6), at eight genomic target sites (Additional file 1: Figure S5, including three sites that have been examined with five tested BEs in Additional file 1: Figure S1) as well as eight target sites where C-to- $\mathrm{T}$ conversions create pathogenic SNVs (Additional file 1: Figure S6, including three sites that have been examined with five tested BEs in Additional file 1: Figure S2). Meanwhile, we also compared these three representative BEs at the same sites in another human cell line U2OS and obtained similar results (Additional file 1: Figures S7, S8).

As BEs can be used to introduce base substitutions to mimic or revert the pathogenic SNVs (Fig. 1), we set up to computationally profile all human pathogenic C-to- $\mathrm{T}$ or T-to-C SNVs to determine which types of BEs might be more suitable for creating or correcting mutations. Twenty BEs with different PAM sequences and editing windows, including the five aforementioned ones, were used for this in silico analysis. The PAM sequences and editing windows of these 20 BEs are listed in Fig. 2a.

For all pathogenic SNVs reported in the NCBI ClinVar database (Fig. 2b), we searched their flanking regions to find nearby PAM sequences that could fit the pathogenic SNV 
a
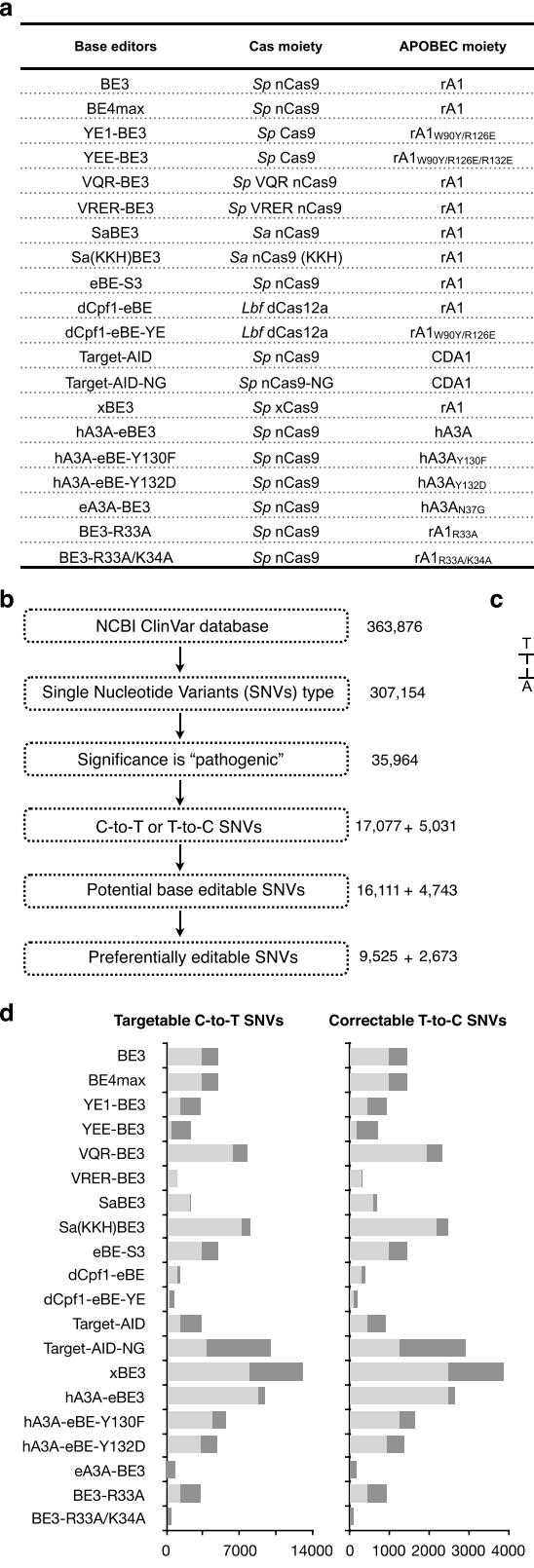

TCTGCAGATCACTGGGGTGG

\begin{tabular}{lll}
$\begin{array}{l}\text { Editing } \\
\text { window }\end{array}$ & $\begin{array}{c}\text { Spacer } \\
\text { length }\end{array}$ & Reference \\
\hline 48 & &
\end{tabular}

1

PAM
NGG
NGG

$4-8 \quad 20$

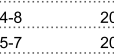

$\begin{array}{ll}5-6 & 20 \\ 4-11 & 20\end{array}$

NGG

NGAN

$4-11$

20

20
20
21

GRRT

$3-12$
$3-12$
$4-8$

21

21

23

$[15$

$[3]$

…...........

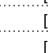

.........

$[3]$

[11]

[5]

PMID: 27492474

PMID: 3016644

PMID: 29512652

\begin{tabular}{cc}
$A 1$ & 1 \\
\hdashline$A$ & $1+3 \times f r e e$
\end{tabular}

$1+3 x$ free

$1+3 x$ free

20

\begin{tabular}{c|c|c|c} 
NGG & $2-13$ & 20 & {$[6]$} \\
\hline NGG & $3-8$ & 20 & {$[6]$}
\end{tabular}

NGG $\quad 2-13$

$\begin{array}{llll}\text { NGG } & 3-8 & 20 & {[6]}\end{array}$

$\begin{array}{llll}\text { NGG } & 4-8(T C) & 20 & {[6]}\end{array}$

$\begin{array}{llll}\text { NGG } & 5-7 & 20 & 40 \\ {[20]}\end{array}$

\begin{tabular}{lll} 
NGG & $5-6(\mathrm{TC})$ & 20 \\
\hline
\end{tabular}

$\left[\begin{array}{l}{[20]} \\ {[20]} \\ {[2]}\end{array}\right.$

$\begin{array}{lll}N:[A, T, C, G] & R:[A, G] & V:[A, C, G]\end{array}$

TCTGCAGATCAĆTGGGGTGGATCCCGAAGGGTCCAT $\frac{1}{A G A C G T C T A G T G A C C C C A C C T A G G G T T C C C A G G A}$

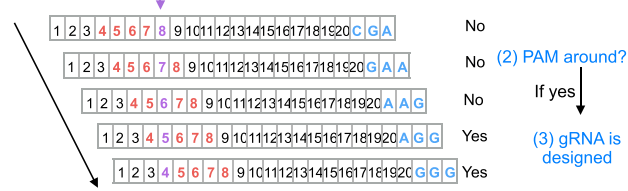

(1) Sliding base editing window around pathogenic SNV

e

\begin{tabular}{|c|c|}
\hline Item & Information \\
\hline Name & NM_005886.2(KATNB1):c.1A>G (p.Met1Val) \\
\hline RS\# (dbSNP) & 879255518 \\
\hline Gene Symbol & KATNB1 \\
\hline Position & Chr16:57737244 \\
\hline PhenotypeIDS & MedGen:C4015525,0MIM:616212 \\
\hline Pheotypelist & Lissencephaly 6, with microcephaly; \\
\hline$\overline{B E}$ & gRNA Spacer + PAM \\
\hline ВЕз & GTGG ACCCTTAGCTGGCTGG \\
\hline BEamax & GTGG ACCCTTCAGCTGGCTGG \\
\hline YE1-ВE3 & none \\
\hline YEE-BE3 & none \\
\hline vQR-BE3 & none \\
\hline VRER-BE3 & none \\
\hline SaBE3 & none \\
\hline $\mathrm{Sa}(\mathrm{KKH}) \mathrm{BE} 3$ & none \\
\hline eBE-S3 & GTGG ACCCTICAGCTGGCTGG \\
\hline dCPf1-eBE & none \\
\hline ACPF1-EBE-YE & none \\
\hline Target-AID & none \\
\hline Target-AID-NG & none \\
\hline XBE3 & TGG AC CTRCAGCTGGCTGG, GTGG ACCCTTCAGCTGGCTG \\
\hline ИA3A-СEE3 & 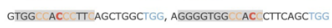 \\
\hline ПАЗА-EBE3-r130F & GGG ACCCTRAGCTGGCIGG \\
\hline ПАЗ3A-EBE3-r132D & none \\
\hline EA3A-BE3 & none \\
\hline BE3-R33А & none \\
\hline BE3-R33A/K34A & none \\
\hline
\end{tabular}

Fig. 2 In silico base editable landscape of pathogenic SNVs. a Summary of 20 selected BEs used in BEable-GPS. PAM, editing window, spacer length, and reference are listed. $\mathbf{b}$ The pipeline for filtering BE editable pathogenic SNVs from NCBI ClinVar database. The numbers of variants are listed in the right for each filtering step. c The workflow of judging whether a pathogenic SNV is potentially targetable. If there are nearby PAM sequences when a SNV is in the editing window, this SNV is considered to be a potentially targetable site by BEs. $\mathbf{d}$ Statistics of number distribution of targetable or preferentially targetable pathogenic SNVs by each BE summarized in $\mathbf{a}$. e The information of one representative pathogenic SNV is provided in the BEable-GPS online website. $\mathbf{f}$ The gRNA spacer region and PAM sequence of each BE for one representative SNV are shown. Pathogenic SNV in red, bystander editing site in yellow, editing window in gray, and PAM in blue

into the editing windows of examined BEs. Based on the existence of PAM sequences, we predicted whether a given SNV could be potentially edited by a specific BE (Fig. 2c). With 20 analyzed BEs, about $94.34 \%$ of 17,077 pathogenic C-to-T SNVs could be generated by at least one $\mathrm{BE}$ to model the relevant genetic disorders and $94.28 \%$ of 5031 pathogenic T-to-C SNVs could be corrected by at least one $\mathrm{BE}$ to examine the potential therapeutic effects. The potentially editable SNVs are summarized in Fig. 2d. The in silico profiling of base editable pathogenic SNVs thus suggests 
broad applications of BEs for human disease study and potential treatment.

To conveniently access the information of these base editable pathogenic point mutations, we constructed a BEable-GPS (http://www.picb.ac.cn/rnomics/BEable-GPS) database for annotation. A "search" function is available to query pathogenic SNVs according to gene symbols, genomic locations or disease phenotypes, and their accessibilities to different BEs (Additional file 1: Figure S9a). With selected BEs, all targetable pathogenic SNVs in queried locations or disease phenotypes can be retrieved in the output list (Additional file 1: Figure S9b). By clicking "Link" button next to a selected SNV, its name (NCBI ClinVar ID), related dbSNP number, chromosome position, gene symbol, related phenotype ID (Fig. 2e), and designed gRNA spacer sequences with the corresponding PAMs highlighted for all applicable BEs (Fig. 2f) are available for further survey.

An online "analysis" function is also available to design specific gRNAs for editable cytosines/guanines from any input sequence (Additional file 1: Figure S10a). Of note, users can also define a specific PAM sequence, editing window, and spacer length to find specific base editable sites for further analysis (Additional file 1: Figure S10a, bottom). All cytosines or guanines that are targetable by the analyzed BEs will be listed together with specific gRNA spacer sequences (Additional file 1: Figure S10b). This online "analysis" function thus expands the application of the BEable-GPS database from pathogenic SNV sites to almost all editable cytosines and guanines. For both search and analysis functions, users can select the union or the intersection of these 20 analyzed BEs for survey and comparison (Additional file 1: Figures S9a, S10a).

It will be of interest for researchers to access BEableGPS and embedded toolsets for their experimental designs to model or correct disease-related mutations. Of note, to reduce substantial off-target mutations, engineered BEs have been continuously developed for precise base editing [20]. We will keep updating this database by including more BEs to provide additional choices for the study of pathogenic mutations and by incorporating off-target prediction to suggest cautions in the future.

\section{Methods}

\section{Cell culture and transfection}

293FT and U2OS cells from ATCC were tested to exclude mycoplasma contamination and not authenticated. For base editing in genomic DNA, 293FT and U2OS cells were seeded in a 24-well plate at a density of $1 \times 10^{5}$ cells/ well and transfected with $250 \mu \mathrm{l}$ serum-free Opti-MEM containing $2.52 \mu \mathrm{l}$ Lipofectamine LTX (Invitrogen/Life Technologies), $0.84 \mu \mathrm{l}$ Lipofectamine Plus (Invitrogen/Life Technologies), $0.5 \mu \mathrm{g}$ BE expression vector (BE3, eBE-S3, BE4max, hA3A-eBE-Y130F or dCpf1-eBE, respectively), and $0.34 \mu \mathrm{g}$ crRNA or sgRNA-expressing plasmid. After $72 \mathrm{~h}$, the genomic DNA was extracted from the cells with QuickExtract DNA Extraction Solution for subsequent analyses.

To generate T-to-C/A-to-G mutations that mimic pathogenic SNV sites individually at the BTK,CLN6, and PGM3 loci, 293FT cells were seeded into a six-well plate at a density of $3 \times 10^{5}$ cells per well and transfected with $250 \mu \mathrm{l}$ serum-free Opti-MEM containing $7.56 \mu \mathrm{l}$ Lipofectamine LTX (Invitrogen/Life Technologies), $2.52 \mu \mathrm{l}$ Lipofectamine Plus (Invitrogen/Life Technologies), $1.5 \mu \mathrm{g}$ ABEmax, and $1.02 \mu \mathrm{g}$ sgRNA-expressing plasmid (sgBTK, sgCLN6 or sgPGM3, respectively). The genomic DNAs of single-cell colonies were individually purified, and ABEmax-created T-to-C mutations were validated by Sanger sequencing (Additional file 2).

\section{Targeted DNA sequencing and data analysis}

Targeted genomic sites were PCR amplified, and an indexed DNA library was prepared for deep sequencing. Indel frequencies were calculated by dividing reads containing at least one inserted and/or deleted nucleotide by all the mapped reads at the same region. Base substitution frequencies were calculated by dividing base substitution reads by total reads.

\section{BE editable analysis of pathogenic SNVs}

The pathogenic mutation sites were downloaded from the NCBI ClinVar database. "Single-nucleotide variants (SNVs)" of "pathogenic" significance were extracted for further analysis. The SNV names including " $\mathrm{C}>\mathrm{T}$ " or " $\mathrm{G}>\mathrm{A}$ " were identified as pathogenic C-to-T/G-to-A SNVs. The SNV names including " $\mathrm{T}>\mathrm{C}$ " or " $\mathrm{A}>\mathrm{G}$ " were identified as pathogenic T-to-C/A-to-G SNVs. The flanking sequence (30 nucleotides upstream and downstream of the SNV site) was extracted from genome sequence according to the coordinate (GRCh38) of SNVs for targetable analysis.

\section{Statistical analysis}

$P$ values were calculated from one-tailed Wilcoxon rank sum test in this study.

\section{Supplementary information}

Supplementary information accompanies this paper at https://doi.org/10. 1186/s13059-019-1839-4.

Additional file 1: Figure S1. Comparison of base editors at overlapped target sites in 293FT cells. Figure S2. Three pathogenic SNVs that can be created by BEs in 293FT cells. Figure S3. Three T-to-C mutations are created by ABEmax in 293FT cells to mimic pathogenic T-to-C/A-to-G SNVs. Figure S4. Comparison of product purity at three ABEmax-generated Tto-C mutations that can be corrected by BEs in 293FT cells. Figure S5. Comparison of base editing outcomes at eight overlapped target sites in 293FT cells. Figure S6. Comparison of base editing outcomes at eight pathogenic SNVs in 293FT cells. Figure S7. Comparison of base editing outcomes at eight overlapped target sites in U2OS cells. Figure S8. 
Comparison of base editing outcomes at eight pathogenic SNVs in U2OS cells. Figure S9. Construction of BEable-GPS website for base editable pathogenic SNVs. Figure S10. Function of gRNA design embedded in the BEable-GPS website.

Additional file 2: Table S1. Oligos used for CBE-gRNA-expressing plasmid construction. Table S2. Oligos used for ABEmax-gRNA-expressing plasmid construction. Table S3. gRNA target sequences and PCR primers for amplifying genomic DNA.

Additional file 3: Table S4. Calculation of indels of base editing results in Fig. 1 and Additional file 1: Figures S1-S4.

Additional file 4: Table S5. Calculation of base substitutions of base editing results in Fig. 1 and Additional file 1: Figures S1-S4.

Additional file 5: Table S6. Calculation of indels of base editing results in Additional file 1: Figures $\mathrm{S} 5-\mathrm{S} 8$.

Additional file 6: Table S7. Calculation of base substitutions of base editing results in Additional file 1: Figure S5-S8.

Additional file 7. Review history.

\section{Acknowledgements}

We like to thank Gordon Carmichael for the critical reading of this manuscript and all lab members for the discussion.

\section{Peer review information}

Yixin Yao was the primary editor on this article and managed its editorial process and peer review in collaboration with the rest of the editorial team.

\section{Review history}

The review history is available as Additional file 7.

\section{Authors' contributions}

LY and JC conceived, designed, and supervised the project. YW performed the bioinformatics analyses with the help of $Y$-CX on database, supervised by LY. RG and JWu performed the experiments with the help of SZ, supervised by JC. JWei prepared libraries for deep sequencing, supervised by LY. LY, BY, and $\mathrm{JC}$ wrote the paper with inputs from all the authors. All authors read and approved the final manuscript

\section{Funding}

This work was supported by grants 2018 YFA070227 (J.C.) and 2018YFC1004602 (J.C.) from MoST and 31730111 (L.Y.), 31822016 (J.C.), 81872305 (J.C.), and 31600654 (J.C.) from NSFC.

\section{Availability of data and materials}

BEable-GPS is available at http://www.picb.ac.cn/rnomics/BEable-GPS. The core source code of BEable-GPS used for gRNA design for pathogenic SNVs are freely available under the MIT license at https://github.com/suduwoniu/ BEable-GPS [21], and an archival version of this code is available on Zenodo with DOI https://doi.org/10.5281/zenodo.3460965 [22]. Targeted deep sequencing datasets are available at the NCBI Gene Expression Omnibus with accession code GSE136749 (https://www.ncbi.n/m.nih.gov/geo/query/acc. cgi?acc= GSE136749) [23] and National Omics Data Encyclopedia with access number NODE: OEP000459 (https://www.biosino.org/node/project/detail/ OEP000459) [24].

Ethics approval and consent to participate

Not applicable.

\section{Consent for publication}

Not applicable.

\section{Competing interests}

The authors declare that they have no competing interests.

\section{Author details}

'CAS Key Laboratory of Computational Biology, CAS-MPG Partner Institute for Computational Biology, Shanghai Institute of Nutrition and Health, Shanghai Institutes for Biological Sciences, University of Chinese Academy of Sciences, Chinese Academy of Sciences, Shanghai 200031, China. ${ }^{2}$ School of Life
Science and Technology, ShanghaiTech University, Shanghai 201210, China. ${ }^{3}$ CAS Center for Excellence in Molecular Cell Science, Shanghai Institute of Biochemistry and Cell Biology, Chinese Academy of Sciences, Shanghai 200031, China. ${ }^{4}$ University of Chinese Academy of Sciences, Beijing 100049, China. ${ }^{5}$ Shanghai Institute for Advanced Immunochemical Studies, ShanghaiTech University, Shanghai 201210, China.

Received: 29 May 2019 Accepted: 27 September 2019

Published online: 23 October 2019

\section{References}

1. Komor AC, Kim YB, Packer MS, Zuris JA, Liu DR. Programmable editing of a target base in genomic DNA without double-stranded DNA cleavage. Nature. 2016;533(7603):420-4

2. Nishida K, Arazoe T, Yachie N, Banno S, Kakimoto M, Tabata M, Mochizuki M, Miyabe A, Araki M, Hara KY, et al. Targeted nucleotide editing using hybrid prokaryotic and vertebrate adaptive immune systems. Science. 2016; 353(6305):aaf8729.

3. Kim YB, Komor AC, Levy JM, Packer MS, Zhao KT, Liu DR. Increasing the genome-targeting scope and precision of base editing with engineered Cas9-cytidine deaminase fusions. Nat Biotechnol. 2017;35(4):371-6.

4. Gehrke JM, Cervantes O, Clement MK, Wu Y, Zeng J, Bauer DE, Pinello L, Joung JK. An APOBEC3A-Cas9 base editor with minimized bystander and off-target activities. Nat Biotechnol. 2018;36(10):977-82.

5. Li X, Wang Y, Liu Y, Yang B, Wang X, Wei J, Lu Z, Zhang Y, Wu J, Huang X, et al. Base editing with a Cpf1-cytidine deaminase fusion. Nat Biotechnol. 2018;36(4):324-7.

6. Wang X, Li J, Wang Y, Yang B, Wei J, Wu J, Wang R, Huang X, Chen J, Yang $L$. Efficient base editing in methylated regions with a human APOBEC3ACas9 fusion. Nat Biotechnol. 2018;36(10):946-9.

7. Yang B, Li X, Lei L, Chen J. APOBEC: from mutator to editor. J Genet Genomics. 2017:44(9):423-37.

8. Yang B, Yang L, Chen J. Development and application of base editors. CRISPR J. 2019;2(2):91-104.

9. Komor AC, Badran AH, Liu DR. CRISPR-based technologies for the manipulation of eukaryotic genomes. Cell. 2017;168(1-2):20-36.

10. Knott GJ, Doudna JA. CRISPR-Cas guides the future of genetic engineering Science. 2018;361(6405):866-9.

11. Wang L, Xue W, Yan L, Li X, Wei J, Chen M, Wu J, Yang B, Yang L, Chen J. Enhanced base editing by co-expression of free uracil DNA glycosylase inhibitor. Cell Res. 2017;27(10):1289-92.

12. Kim K, Ryu SM, Kim ST, Baek G, Kim D, Lim K, Chung E, Kim S, Kim JS. Highly efficient RNA-guided base editing in mouse embryos. Nat Biotechnol. 2017; 35(5):435-7.

13. Liu Z, Lu Z, Yang G, Huang S, Li G, Feng S, Liu Y, Li J, Yu W, Zhang Y, et al. Efficient generation of mouse models of human diseases via ABE- and BEmediated base editing. Nat Commun. 2018;9(1):2338

14. Li J, Liu Z, Huang S, Wang X, Li G, Xu Y, Yu W, Chen S, Zhang Y, Ma H, et al. Efficient base editing in $\mathrm{G} / \mathrm{C}$-rich regions to model androgen insensitivity syndrome. Cell Res. 2019:29:174-6.

15. Koblan LW, Doman JL, Wilson C, Levy JM, Tay T, Newby GA, Maianti JP, Raguram A, Liu DR. Improving cytidine and adenine base editors by expression optimization and ancestral reconstruction. Nat Biotechnol. 2018; 36(9):843-6

16. Ito F, Fu Y, Kao SA, Yang H, Chen XS. Family-wide comparative analysis of cytidine and methylcytidine deamination by eleven human APOBEC proteins. J Mol Biol. 2017:429(12):1787-99.

17. Lei L, Chen H, Xue W, Yang B, Hu B, Wei J, Wang L, Cui Y, Li W, Wang J, et al. APOBEC3 induces mutations during repair of CRISPR-Cas9-generated DNA breaks. Nat Struct Mol Biol. 2018;25(1):45-52.

18. Landrum MJ, Lee JM, Benson M, Brown G, Chao C, Chitipiralla S, Gu B, Hart J, Hoffman D, Hoover J, et al. ClinVar: public archive of interpretations of clinically relevant variants. Nucleic Acids Res. 2016;44(D1):D862-8.

19. Rees HA, Liu DR. Base editing: precision chemistry on the genome and transcriptome of living cells. Nat Rev Genet. 2018;19:770-88.

20. Grünewald J, Zhou R, Garcia SP, Iyer S, Lareau CA, Aryee MJ, Joung JK Transcriptome-wide off-target RNA editing induced by CRISPR-guided DNA base editors. Nature. 2019:569:433-7.

21. Wang Y, Gao R, Wu J, Xiong Y, Wei J, Zhang S, Yang B, Chen J, Yang L. BEable-GPS: gRNA design tools for pathogenic SNVs. GitHub Repository. https://github.com/suduwoniu/BEable-GPS. Accessed 25 Sept 2019. 
22. Wang Y, Gao R, Wu J, Xiong Y, Wei J, Zhang S, Yang B, Chen J, Yang L. BEable-GPS: gRNA design tools for pathogenic SNVs. Zenodo Respository. https://doi.org/10.5281/Zenodo.3460965. Accessed 25 Sept 2019.

23. Yang L, Chen J, Yang B, Wang Y, Gao R, Wu J, Xiong Y, Wei J, Zhang S.

Comparison of cytosine base editors and development of the BEable-GPS database for targeting pathogenic SNVs. Gene Expression Omnibus. https:// www.ncbi.nlm.nih.gov/geo/query/acc.cgi?acc=GSE136749. Accessed 25 Sept 2019.

24. Wang Y, Gao R, Wu J, Xiong Y, Wei J, Zhang S, Yang B, Chen J, Yang L. Comparison of cytosine base editors and development of the BEable-GPS database for targeting pathogenic SNVs. The National Omics Data Encyclopedia (NODE). https:/www.biosino.org/node/project/detail/ OEP000459. Accessed 25 Sept 2019

\section{Publisher's Note}

Springer Nature remains neutral with regard to jurisdictional claims in published maps and institutional affiliations.

Ready to submit your research? Choose BMC and benefit from:

- fast, convenient online submission

- thorough peer review by experienced researchers in your field

- rapid publication on acceptance

- support for research data, including large and complex data types

- gold Open Access which fosters wider collaboration and increased citations

- maximum visibility for your research: over $100 \mathrm{M}$ website views per year

At BMC, research is always in progress.

Learn more biomedcentral.com/submissions 